\section{Catastrophic Antiphospholipid Syndrome and Polyarteritis Nodosa}

\section{To the Editor:}

The catastrophic antiphospholipid syndrome (CAPS) is a rare and severe manifestation of the antiphospholipid syndrome (APS $)^{1}$. We describe a case with features of both CAPS and polyarteritis nodosa (PAN), described only once previously in the literature.

A healthy 66-year-old man presented to an outside institution with progressive bilateral myalgias in his arms and lower legs. He reported fatigue and a 10-lb unintentional weight loss over the preceding month, but denied other symptoms or exposures. Blood pressure was 140/91 mm Hg, elevated from 120/70 mm Hg recorded 3 weeks earlier. He had cool, pale feet with diminished sensation and limited range of motion. Popliteal pulses were palpable, but dorsalis pedis and posterior tibial pulses were not detectable by Doppler ultrasound. The remainder of the examination was normal.

Hemoglobin was $109 \mathrm{~g} / \mathrm{l}$ with mean corpuscular volume $87 \mathrm{fl}$, white blood cell count $31.6 \times 10^{9} / 1$, (neutrophils $29 \times 10^{9} / 1$ ), and platelets $967 \times$ $10^{9} / 1$. Albumin was $29 \mathrm{~g} / \mathrm{l}$ and creatinine $71 \mathrm{mmol} / \mathrm{l}$. Coagulation measures were normal. Lupus anticoagulant was positive. Testing was done by an activated partial thromboplastin time assay, mixing studies, and a dilute Russell venom viper test, and confirmatory testing was performed. Aspartate aminotransferase was $107 \mathrm{U} / 1$, alanine aminotransferase (ALT) 142 U/l, gamma-glutamyl transferase 52 U/l, and alkaline phosphatase 152 U/l. Creatine kinase was $1317 \mathrm{U} / 1$ and lactate dehydrogenase $410 \mathrm{U} / \mathrm{l}$. Urinalysis was bland. Erythrocyte sedimentation rate (ESR) was $93 \mathrm{~mm} / \mathrm{h}$, C-reactive protein (CRP) $164 \mathrm{mg} / \mathrm{l}, \mathrm{C} 3 \quad 1.86 \mathrm{~g} / \mathrm{l}$, and C4 $0.31 \mathrm{~g} / \mathrm{l}$.
Antinuclear antibodies, antineutrophil cytoplasmic antibodies, rheumatoid factor, hepatitis B, hepatitis C serology, and HIV serology were negative.

Computed tomography (CT) angiogram showed bilateral occlusions of the peroneal, anterior, and posterior tibial arteries and bilateral hypoattenuating areas in the cortices of the kidneys, consistent with infarction. The patient was started on pulse intravenous (IV) methylprednisolone, cyclophosphamide, and unfractionated heparin. Seven days later he had no pain in his feet and Doppler-detectable peripheral pulses. The results of the lupus anticoagulant test were not available at the time and heparin was discontinued. He was discharged taking prednisone $60 \mathrm{mg}$ daily and $75 \mathrm{mg}$ of oral cyclophosphamide.

He was readmitted 3 weeks later with worsening pain in both feet as well as paresthesias and pain of both upper limbs. He was restarted on pulse IV methyprednisolone and IV heparin and transferred to our institution. He reported an additional 20-lb unintentional weight loss over the preceding month. Examination showed bilateral erythema of both feet and necrosis of the dorsal surface of the left foot with no palpable distal pulses and a cyanotic and cool left hand with a weak radial pulse. ESR was 102 $\mathrm{mm} / \mathrm{h}$, CRP $5.4 \mathrm{mg} / \mathrm{l}$, and ALT was persistently elevated at $94 \mathrm{U} / \mathrm{l}$. Urinalysis revealed $3+$ blood and 1+ protein, and a 24-h urine collection demonstrated $2.08 \mathrm{~g}$ protein.

He underwent emergency bilateral below-knee amputations. Biopsy revealed gangrenous changes and occlusion of the dorsalis pedis and popliteal arteries with intravascular thrombosis. Repeat CT angiogram showed an aneurysm of the right main renal artery and the previously visualized bilateral renal cortical infarcts. The brachial artery was occluded and severely narrowed and the internal carotid arteries were narrowed bilaterally (Figure 1). Angiography revealed narrowing of the left brachial artery

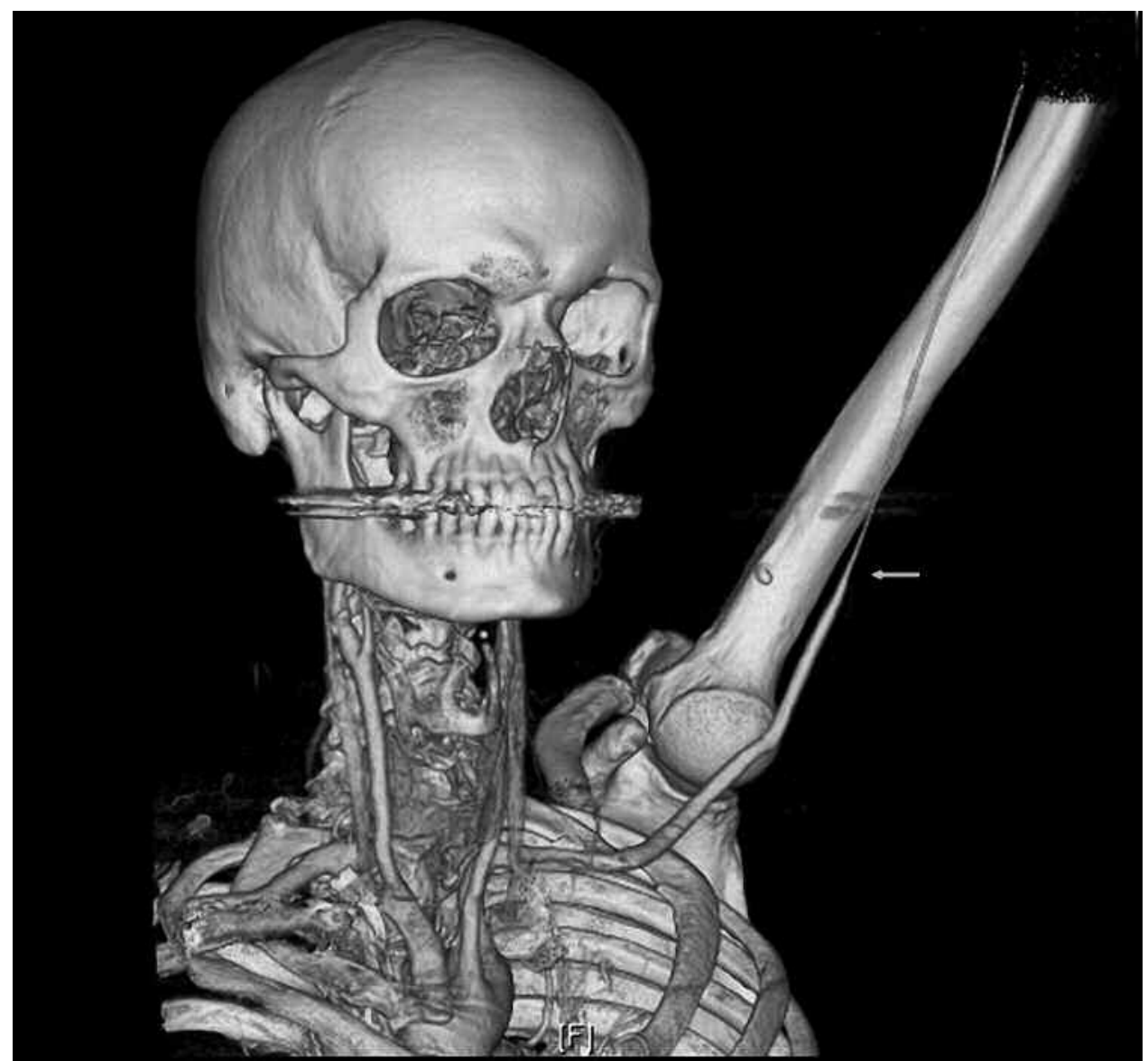

Figure 1. Computed tomography angiogram 3-dimensional reconstruction shows narrowing of left brachial artery. 
with an occlusion at its bifurcation. There was aneurysmal dilatation of the right renal artery as well as ectasia of celiac artery, narrowing of left hepatic artery, and focal segmental occlusions of the superior mesenteric artery (Figure 2). Brain magnetic resonance imaging (MRI)/MR angiography showed bilateral ischemic changes in the subcortical white matter and deeper white matter. There was no evidence of aneurysms. Repeat lupus anticoagulant testing performed 10 weeks after the first result was positive. The patient's course was complicated by the development of neutropenia and multifocal pneumonia, requiring ventilation. He developed a gluteal hematoma requiring multiple transfusions and temporary cessation of anticoagulation. He ultimately recovered and was discharged to rehabilitation.

CAPS differentiates an accelerated form of the APS that results in widespread thrombotic disease and multiorgan failure. Recent animal models have suggested that complement activation may mediate induction of thrombosis and activation of endothelial cells in $\mathrm{APS}^{2}$. Diagnosis requires evidence of involvement of several organ systems, confirmation by histopathology of small-vessel occlusion, laboratory confirmation of the presence of antiphospholipid antibodies, and the development of manifestations in a short time ${ }^{3}$.

The optimal management of CAPS is not known due to the rarity of the condition and the lack of controlled studies. However, prompt clinical recognition and treatment may lead to improved outcomes ${ }^{4}$. Goals usually include treatment of precipitating factors, suppression of the systemic inflammatory response syndrome, and anticoagulation ${ }^{5}$. IV heparin is usually administered followed by longterm oral anticoagulation. Clinical worsening after the cessation of heparin emphasizes the importance of continued anticoagulation when CAPS is suspected. Attempts to reduce antiphospholipid antibody titers with plasma exchange or administration of IV immunoglobulins have shown promise in recent case series ${ }^{6}$.
Cyclophosphamide has shown efficacy in systemic lupus erythematosus-associated $\mathrm{CAPS}^{7}$. In individuals with severe thrombocytopenia, B cell depletion with rituximab has been successful ${ }^{8}$

On re-presentation to our institution, initial management involved reinitiation of anticoagulation, broad-spectrum antibiotics, and prompt leg amputations. Steroids and cylophosphamide were continued and plasma exchange was not undertaken because of concerns regarding the potential hazards of further immunosuppression.

PAN, a systemic necrotizing vasculitis affecting the medium-size arteries, is diagnosed when 3 of 10 criteria are present. Features in this case included significant weight loss, myalgias with weakness, new-onset hypertension, renal infarcts, renal aneurysms, and elevated ESR and CRP. The combination of renal aneurysms and renal cortical infarcts on CT is highly suggestive of $\mathrm{PAN}^{9}$. If severe, the usual treatment is with glucocorticoids and cyclophosphamide, as in this case.

Our case has features of both CAPS and PAN, reported only once in the literature ${ }^{10}$. That report described a 44 -year-old man who had angiographic evidence of intraabdominal aneurysms as well as renal and central nervous system infarction. He had positive rapid plasma reagin and $\mathrm{T}$ pallidum antibody tests, as well as a positive anticardiolipin antibody and antiphosphatidyl serine antibody, but no histopathologic confirmation of thrombosis. Anticoagulation was not initiated and he died despite treatment with broad-spectrum antibiotics, methylprednisolone, and cyclophosphamide.

The decision to undertake anticoagulation requires great care because of the risks of bleeding from aneurysms. Our patient had a significant gluteal bleed soon after widespread thrombosis. In addition, while being treated with immunosuppression, he developed a severe multifocal pneumonia. We highlight the difficulties in clinical decision-making that arise in the management of this complex syndrome.

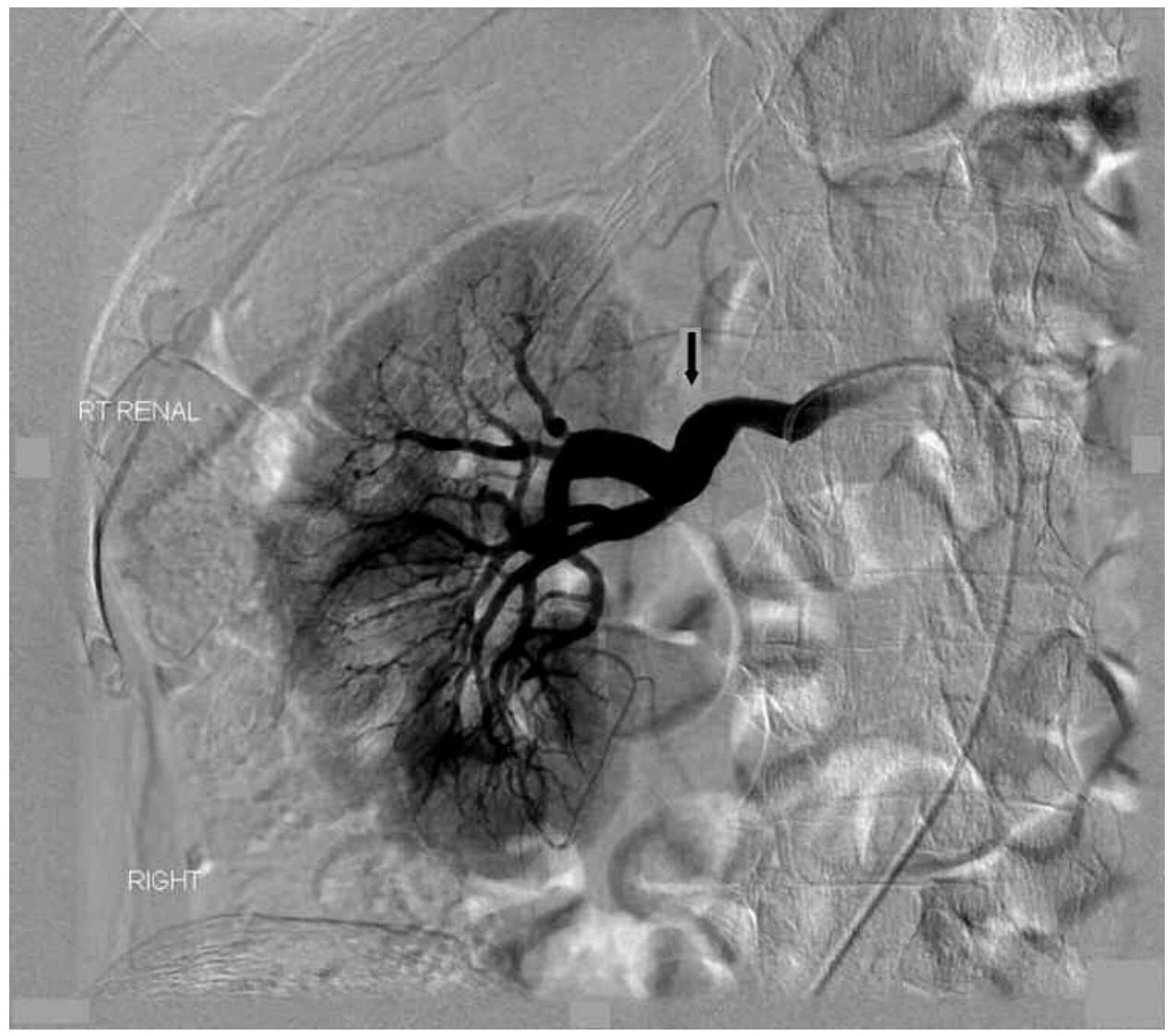

Figure 2. Right renal angiogram shows multiple microaneurysms as well as aneurysmal dilatation of the right renal artery. 
SAUL MILLER, MD; ELI MILLER, BSc, Department of Internal Medicine; NADER KHALIDI, MD, FRCPC, Department of Rheumatology, McMaster University, 240 James Street South, Hamilton, Ontario L8P 3B3, Canada. Address correspondence to Dr. Khalidi;

E-mail: naderkhalidi@sympatico.ca

\section{REFERENCES}

1. Cervera R, Piette JC, Font J, Khamashta MA, Shoenfeld Y, Camps MT, et al. Antiphospholipid syndrome: clinical and immunologic manifestations and patterns of disease expression in a cohort of 1,000 patients. Arthritis Rheum 2002;46:1019-27.

2. Pierangeli SS, Girardi G, Vega-Ostertag M, Liu X, Espinola RG, Salmon J. Requirement of activation of complement C3 and C5 for antiphospholipid antibody-mediated thrombophilia. Arthritis Rheum 2005;52:2120-4.

3. Asherson RA, Cervera R, de Groot PG, Erkan D, Boffa MC, Piette JC, et al. Catastrophic antiphospholipid syndrome: international consensus statement on classification criteria and treatment guidelines. Lupus 2003;12:530-4.

4. Asherson RA. Multiorgan failure and antiphospholipid antibodies: the catastrophic antiphospholipid (Asherson's) syndrome. Immunobiology 2005;210:727-33.
5. Asherson RA, Cervera R. Catastrophic antiphospholipid syndrome Curr Opin Hematol 2000;7:325-9.

6. Cervera R, Bucciarelli S, Plasin MA, Gomez-Puerta JA, Plaza J, Pons-Estel G, et al. Catastrophic antiphospholipid syndrome (CAPS): descriptive analysis of a series of 280 patients from the “CAPS Registry". J Autoimmun 2009;32:240-5.

7. Bayraktar UD, Erkan D, Bucciarelli S, Espinosa G, Asherson R. The clinical spectrum of catastrophic antiphospholipid syndrome in the absence and presence of lupus. J Rheumatol 2007;34:346-52.

8. Rubenstein E, Arkfeld DG, Metyas S, Shinada S, Ehresmann S, Liebman HA. Rituximab treatment for resistant antiphospholipid syndrome. J Rheumatol 2006;33:355-7.

9. Wilms G, Oyen R, Waer M, Baert AL, Michielsen P. CT demonstration of aneurysms in polyarteritis nodosa. J Comput Assist Tomogr 1986;10:513-5.

10. Han BK, Inaganti K, Fahmi S, Reimold A. Polyarteritis nodosa complicated by catastrophic antiphospholipid syndrome. J Clin Rheumatol 2004;10:210-3.

J Rheumatol 2009;36:11; doi:10.3899/jrheum.090301 